\title{
Control interno y su incidencia en la rentabilidad de empresa cobros del norte S.A
}

Fátima Johana Paiva Acuña ${ }^{1}$

1 Egresada de la maestría en contabilidad con énfasis en auditoria UNAN-Managua FAREM-Estelí. Correo electrónico: fpaiavaa@yahoo.es

\section{RESUMEN}

Esta investigación se evalúa en control interno y su incidencia en la rentabilidad de la Empresa Cobros del Norte S. A En el Periodo 2011 y aportar recomendaciones con las cuales pueden mejorar. El tipo de estudio es descriptivo y de corte transversal porque se está evaluando un periodo siendo este el 2011. El enfoque es cualitativo con técnicas cuantitativas y cualitativas. Se aplicaron entrevistas y encuestas, se reforzó con investigación documental para confirmar la veracidad de la información brindada. La investigación se elaboró en base a los componentes del modelo COSO I y la rentabilidad se basa en el análisis Costo-Beneficio.

Según la problemática encontrada se corrobora que el control interno es deficiente analizando las grandes debilidades encontradas durante el proceso empezando desde el ambiente de control de la empresa, siendo uno de los principales problemas la mala segregación de funciones y la falta de información financiera a tiempo. Se recomienda tomar medidas correctivas para solucionar las debilidades, en primer lugar la elaboración de un manual de procedimientos, un manual de funciones donde se establezcan las líneas de autoridad y responsabilidad, y la supervisión constante del proceso de compras.

Palabras claves: control interno, rentabilidad, Elementos del COSO, Costo-Beneficio

\section{ABSTRACT}

This research evaluates internal control and its impact on the profitability of the Company Collections North S. A In the period 2011 and provide recommendations that can improve. The type of study is descriptive and cross-sectional because it is evaluating a period 2011. El being the qualitative approach is quantitative and qualitative techniques because interviews are done analytically which are processed according to the assessment of the functioning of control internal surveys and processed quantitatively represented in percentages according to the sample, was reinforced with documentary research to confirm the accuracy of the information provided.

Specifically the investigation is prepared using the components of the COSO model I, and profitability is based on cost-benefit analysis. According to the problems encountered is confirmed that the internal control is deficient analyzing the great weaknesses found during the process starting from the control environment of the company, one of the main problems of poor segregation of duties, and lack of timely financial information is recommended to take remedial action to address the weaknesses found, first developing a procedures manual, a manual functions where established lines of authority and responsibility, and to perform continuous monitoring of the procurement process

Keywords: internal control, profitability, Elements of coso, Cost-Benefit 


\section{INTRODUCCIÓN}

La evaluación del sistema de control interno, por medio de los manuales de procedimientos, afianza las fortalezas de las empresas frente a la gestión. En razón de esta importancia es que adquiere el sistema de control interno para cualquier entidad, se hace necesario hacer el levantamiento de procedimientos actuales, los cuales son el punto de partida y el principal soporte para llevar a cabo los cambios que con tanta urgencia se requieren para alcanzar y ratificar la eficiencia, efectividad, eficacia y economía en todos los procesos.

El control interno, es una necesidad administrativa de todo ente económico, si consideramos, que en toda empresa existe la posibilidad de contar con empleados deshonestos, descuidados o ineficientes, que hacen que la custodia de los activos sea deficiente, que la ejecución del cumplimientos de funciones no se efectué apropiadamente y que se genere información confiable sobre la cual se puedan fundamentar decisiones, este estudio centra su atención Evaluación del Control interno y su incidencia en la rentabilidad de la Empresa Cobros del Norte S. A (ECONORTE) en el periodo 2011.

ECONORTE, surge en el 2003, constituida como una sociedad anónima, para brindar el servicio cobro de facturas de Energía en el territorio Norte del País, como contratista de la Trasnacional Gas Natural (Disnorte), actualmente está prestando también los servicios de Atención al Cliente.

La investigación está enmarcada dentro del ámbito de control, específicamente en los puntos de recaudo ubicados en los municipios de Sébaco, Ocotal y Somoto. Para la realización de este trabajo se consultaron estudios vinculados con el tema de estudio, Este problema no ha sido tratado con el mismo enfoque, pero si con temas relacionados tales como: (Álvarez, 2002 realiza una evaluación del control interno basado en el modelo COSO, en esta investigación se integran los cinco componentes de evaluación del COSO, como método de evaluación del control interno de una empresa en particular, este modelo es idóneo para medir la eficiencia y eficacia de las áreas de una empresa. (Reyes, 2001), realiza una evaluación del control interno basado en el modelo COSO, en esta investigación se integran los cinco componentes de evaluación del COSO

ECONORTE durante su existencia no ha realizado ningún trabajo investigativo ni tampoco se ha evaluado su control interno. Con esta investigación, se reconoce la importancia del control interno dentro de las empresas y su influencia en el buen andar de las finanzas. Un buen sistema de control interno, permite un mejor nivel operativo y por consiguiente trasciende en los resultados.

Esta investigación surge por la necesidad de brindar a esta empresa un medio estratégico que contribuya al desarrollo efectivo de la planeación organizacional, permitiendo con ello esquematizar cada factor que influya posiblemente para que la empresa pueda optimizar sus funciones. Por otro lado, es de gran relevancia hacer notar que el Sistema de Control Interno que se evaluará, va a ser objeto de fortalecimiento para que la empresa disponga de otra herramienta que le permita desarrollar su planeación tomando en cuenta los factores que constituyen la misma, según sea las funciones que ejerce cada empleado apoyándose para ello del resultado final de la investigación.

El propósito de esta investigación, permite enfocar una visión especifica de lo que se quiere alcanzar, para mejorar las funciones del proceso administrativo y contable, las funciones de cada empleado que labora en la empresa con la finalidad de mejorar las debilidades, a la vez que sirva de aporte aquellas empresa que no cuentan con un adecuado sistema de control interno, el cual es importante, en virtud de que 
las empresas día a día presentan mayor desarrollo y solo mediante la obtención de su máxima eficiencia operativa podrán mantenerse firmes y sólidas dentro de un marco de competencia.

En los últimos años, ECONORTE ha tenido tres fraudes por parte del personal que está ubicado en los puntos de recaudo y en los centros de atención al cliente, esto debido a la falta de un control interno porque no tienen una persona destinada a dar seguimiento a estas actividades. El exceso de confianza por parte de la gerencia, quien está a cargo de toda la empresa no se ha encargado de delegar sus funciones, ha llevado a que la empresa durante los últimos años no haya logrado obtener las ganancias esperadas.

La importancia de tener un buen sistema de control interno en las organizaciones, se ha incrementado en los últimos años, esto debido a lo práctico que resulta al medir la eficiencia y la productividad al momento de implantarlos; en especial si se centra en las actividades básicas que se realizan, pues de ello dependen mantener una contabilidad confiable.

ECONORTE actualmente, no tiene un modelo o procedimiento específico de cómo debe ser un sistema de control interno, sin embargo existen lineamientos mínimos (cartas, memorandos, reglamento interno), que contribuyen al cumplimiento de sus objetivos, por lo tanto se requiere implantar un modelo de control interno administrativo y contable que garantice la eficiencia y economía de las operaciones de la empresa, facilitando la correcta ejecución de las funciones y asegurando la oportunidad, claridad, utilidad y confiabilidad de la información administrativa y contable.

Como objetivo principal se planteo Evaluar el control interno y su incidencia en la rentabilidad de ECONORTE. en el municipio de Estelí, en el periodo 2011, utilizando como modelo de referencia COSO para el control interno y para evaluar la rentabilidad se utilizo Beneficio / Costo.

\section{MATERIAL Y MÉTODO}

Para realizar la investigación se tomo como referencia el Modelo COSO, Según Mantilla (2003, p4.), El informe del "Committe of sponsoring "(COSO) define al control interno como un proceso llevado a cabo por las personas de una organización, diseñado con el fin de proporcionar un grado de seguridad "razonable" para la consecución de sus objetivos, dentro de las siguientes categorías:

- Eficiencia y eficacia de la operatoria

- Fiabilidad de la información financiera

- Cumplimiento de las leyes y normas aplicables

El control interno comprende el plan de organización y todos los métodos y medidas coordinadas adoptadas dentro de una empresa para salvaguardar sus bienes, comprobar la exactitud y veracidad de los datos contables, promover la eficiencia operante y estimular la adhesión a los métodos prescritos por la gerencia (Mantilla, 2003).

El control interno es un proceso efectuado por el consejo de administración, la dirección y el resto de personal de una entidad, diseñado con el objeto de proporcionar un grado de seguridad razonable en cuanto a la consecución de objetivos dentro de las tres siguientes categorías:

- Efectividad y eficiencia de las operaciones.

- Suficiencia y confiabilidad de la información financiera.

- Cumplimiento de las leyes y regulaciones aplicable.

Rentabilidad es una noción que se aplica a toda acción económica en la que se movilizan unos medios, materiales, humanos y financieros con el fin de obtener unos resultados. (López Joaquín y Otros 
2001) "Esto supone la comparación entre la renta generada y los medios utilizados para obtenerla con el fin de permitir la elección entre alternativas o juzgar la eficiencia de las acciones realizadas, según que el análisis realizado sea a priori o a posteriori".

El análisis de costo-beneficio es una técnica importante dentro del ámbito de la teoría de la decisión. Pretende determinar la conveniencia de proyecto mediante la enumeración y valoración posterior en términos monetarios de todos los costos y beneficios derivados directa e indirectamente de dicho proyecto. Este método se aplica a obras sociales, proyectos colectivos o individuales, empresas privadas, planes de negocios, etc., prestando atención a la importancia y cuantificación de sus consecuencias sociales y/o económicas.

Se tomaron las siguientes variables para el estudio:

\section{Control Interno}

Los componentes del control interno según el modelo coso

- Ambiente de Control

- Evaluación de Riesgos

- Actividades de Control

- Información y Comunicación

- Supervisión

\section{Rentabilidad}

\section{- Análisis Costo Beneficio}

Se estableció un enfoque cuali-cuantitativo, debido que se estableció una muestra reducida de investigación, y se abordan tanto técnicas cualitativas como los análisis en las entrevistas como también cuantitativas en las encuestas.

El estudio a utilizar para esta investigación fue de tipo inductivo ya que se parte de hechos particulares ocurridos en ECONORTE, donde se enmarca el problema de investigación, para llegar a conclusiones.
Se plantea de que la inducción es ante todo una forma de raciocinio o argumentación, por lo que lleva un análisis ordenado, coherente y lógico del problema de investigación, tomando como referencia premisas verdaderas, por lo que sus conclusiones estarán relacionadas con sus premisas como el todo lo está con las partes.

\section{TIPO DE INVESTIGACIÓN}

Se evaluó el control interno que está implementando ECONORTE área de contabilidad y centros de pagos, mediante el tipo de estudio descriptivo, el cual consiste en describir un fenómeno sometiéndolo a una evaluación e identificando las debilidades encontradas para recomendar su mejora.

Este tipo de estudio se vale de técnicas convenientes para investigaciones in situ por lo que se utilizaron técnicas encuestas y entrevistas individuales para cada uno de los integrantes de la muestra, esto para obtener una respuesta veras y un punto de vista de cada uno de los que integran la junta directa, Administradora, contador y las Cajeras de los puntos de Recaudos, la información que resultó.

\section{Investigación documental}

En la investigación establecieron comportamientos concretos y actitudes del personal en la cual se descubre y comprueba la asociación entre las variables establecidas de investigación, de acuerdo con los objetivos planteados. El diseño de investigación que se utilizó el no experimental, de corte transversal porque se evaluó solo un periodo establecido el año 2011.

\section{Población y Muestra}

La población que aplica el desarrollo de esta investigación es de 15 personas, que laboran en ECONORTE, ya que ellos pueden proporcionar información valiosa, retroalimentación básica para completar los objetivos de la investigación 
Asimismo, la información que los empleados proporcionarán servirá de parámetro, para identificar en el proceso de cobros en qué parte del mismo existen fallas, si existe algún paso o proceso mal ejecutado, si se está cumpliendo con la gestión de cobro y la entrega en tiempo a la trasnacional

La investigación tomará como muestra a ECONORTE. De esta forma se está utilizando una muestra no probabilística por los aspectos técnicos que están en juego:
a) Presidente de la Empresa
b) 7. Socios
c) 1. Responsable de Contabilidad
d) 4. Cajeras de Puntos Fijos

\section{RESULTADOS Y DISCUSIÓN}

\section{Control Interno}

Según la información recopilada en el trabajo de campo se revela que no existe un código de ética escrito, sin embargo ellos mencionaron que desde el momento en que el trabajador entra a laborar se establecen los valores éticos de manera verbal y se hace referencia al Código Ético que tiene el contratante Disnorte, aun así $100 \%$ contestó que los trabajadores responden adecuadamente a la integridad y los valores éticos los entrevistados también hicieron referencia a los valores de Compromiso, Respeto y Calidad diciendo que eran los más importantes.

Se comprueba que no existe un manual de funciones escrito, simplemente se establecen las funciones y no se realiza un contrato de trabajo formal a como lo establece la Ley, por lo que no existe una guía que apoye a los trabajadores a saber con exactitud cuáles son las funciones por las cuales tiene que responder,

La segregación de funciones se proyectan un 100\% en los puntos de recaudo, si se cumplen las funciones asignadas sin embargo, el administrador realiza algunas funciones de la contabilidad, hay una mala segregación de funciones, se corre el riesgo de fraude por la dependencia de una persona en todo el ciclo, Según un $92 \%$ de los encuestados opina que tienen definidas las responsabilidades, y también son conocidas por su responsable inmediato.

Según los encuestados en un $100 \%$ contestan que las decisiones que se toman son aprobadas por la junta directiva y se dejan en el libro de actas, sin embargo no se tiene un manual de funciones escrito donde se establezca las responsabilidades de cada cargo en la Empresa, por lo que se podría decir que las actividades de aprobación se realizan de manera empírica ya que no hay nada escrito que respalde el buen seguimiento de la autorización.

EI 92\% de los a encuestados contestan que no existe un organigrama en la Empresa que sea de conocimiento de los empleados en este caso hay una debilidad en el ambiente de control ya que un organigrama define claramente los niveles de autoridad de cada empleado y deberá ir acompañado de un manual en el que se asigne las relaciones jerárquicas y funcionales para cada cargo, por lo que se recomienda su elaboración para fortalecer el control interno de la Empresa.

Se consulta a los encuestados si ellos realizan solo las funciones destinadas a su cargo y en un $33 \%$ contestan que no realizan solo las funciones que se les asigno, dado que la falta de personal hace que ellos tengan que hacer varias cosas fuera de sus funciones, sin embargo considera un $89 \%$ que hay una buena coordinación en las áreas de trabajo.

Al preguntar sobre el conocimiento de la misión, visión y valores en un $89 \%$ contestan que no la conocen, sin embargo la Junta directiva dice que se tiene una misión y una visión definida por lo que se le encomendó la tarea al administrador de que la de a conocer a todos los empleados, siendo una empresa en crecimiento es importante saber que hacen y 
a donde se dirigen guiando al resto de personal a alcanzar la meta que desea sobre todo para cumplir con las metas propuesta por su contratante.

En toda empresa deberá de existir un organigrama y deberá ser de conocimiento de todos los empleados, esto con el fin de fortalecer el control interno de las empresas.

Se investigó a través de una entrevista al personal de los centros de pagos y ellos informan que el mayor riesgo es de Robo y/o Asalto a la persona encargada del traslado de valores ya que este se realiza sin ninguna seguridad y siempre se hace a la misma hora

En lo que respecta a factores internos respondieron que estos no son tomados ya que la Administración según los resultados tienen una probabilidad alta de riesgos, como puede ser un auto robo por parte del personal y exceso de confianza ya que nunca se le arque al personal de los centros de pagos.

El $100 \%$ de los encuestados informa que no existe un manual de procedimiento, que se aplique en el área de caja, sin embargo ellos informan que el contratante es quien se encargada de entrenarlos pues ellos se rigen por el procedimiento que tiene establecido, el cual se comprobó se cumple con el mismo, sin embargo la administración nunca ha revisado si se aplica correctamente.

Con respecto a los depósitos diario estos si se cumplen en un $89 \%$ pues al contratante se le debe de entregar diario, pero la administración no se da cuenta de esta actividad, se le recomienda a la Administración llevar un control diario de reporte de depósito y solicitar al contratante la confirmación de los mismos.

El $100 \%$ de personal de caja encuestado informa que existe y conocen el procedimiento de caja pero este es del contratante y como empresa ellos no tienen definido un procedimiento, por lo cual desconocen si la empresa tiene uno propio.

En la encuesta se preguntó que si están debidamente documentadas todas las decisiones importantes de la empresa? en un $100 \%$ contestaron que sí, las decisiones más importantes son tomadas por la junta directiva las cuales se dejan registradas en el libro de actas y caso no estar todos de acuerdo se realiza por votación, aunque algunas decisiones son tomadas directamente por el presidente de la junta, esto trae consigo que varias operaciones importantes estén concentradas en una sola persona lo que podría traer consigo actos ilícitos al tener la plena libertad de manejar todo un proceso.

De la misma manera se preguntó de manera directa si la misma persona que hace los cheques es la misma que los registra, según la administradora ella es la responsable de elaboración y del registro pues no tiene un contador por lo que casi siempre hace la función de contador por lo que se deduce que si se está dando esta situación, no tiene por qué aprobarse y registrarse las transacciones por la misma persona. El $100 \%$ señala que no existe un manual de registro de las actividades financieras que se hace manera empírica por la experiencia.

Según la muestra tomada para el análisis obtuvimos un $100 \%$ de respuesta de que se mantiene un archivo adecuado de la información.

Se le preguntó a los encuestados ¿Los estados financieros son elaborados de forma mensual? El $100 \%$ contestó que lo estados financieros no son elaborados de forma mensual, pues los socios que pertenecen a la junta directiva retrasan la información y que se realizan anual cuando se tiene que realizar el pago de los impuestos, sin embargo al momento que se elabora están con todos sus soportes y cumplen con las norma vigentes del país. 
Se preguntó a los entrevistados ¿Cuáles son los canales de comunicación dentro de la empresa?, quien los define y se considera efectivos, según encuesta realizada se logró identificar que la información es debidamente comunicada al personal de la empresa pero en su mayoría por el contratante y no por la administración, ellos exponen que al no tenerlos en sus oficinas se le hace un poco complicado la comunicación, sin embargo se tienen canales definidos como el correo electrónico y el teléfono, y la mayoría de la Junta directiva al menos 3 veces a la semana visita los centros de pagos donde se les da información

Comentaron los encuestados que los canales de comunicación los consideran adecuados, dado que no han tenido problemas por falta de comunicación entre las áreas, por lo que consideran que la comunicación es adecuada.

Se les preguntó que si la Gerencia estaba interesada en comunicar los valores éticos, los objetivos y las políticas y según respondieron en su mayoría que si estaban interesados, sin embargo no se encontró en libro de actas si se tiene previsto en qué fecha se realizaría

En cuanto a los egresos todos están soportados por la Administración sin embargo existe un retraso por parte de la Junta directa, lo que hace que los estados financieros no están a tiempo, la empresa no realiza ningún arqueo en los puntos de recaudo, no existe persona contratado para esta actividad.

Existe riesgo ya que no se elaboran oportunamente las conciliaciones bancarias. Adicionalmente la persona encargada de realizar la misma, tiene dentro de su responsabilidad el registro de los depósitos y cheques emitidos. No hay segregación de funciones en cuanto a cada una de las actividades descritas anteriormente. Se verificó la existencia del fondo de caja chica. No se realizan arqueos sorpresivos, no hay formatos pre-definidos para liquidar los gastos, no hay tiempo límite para la liquidación de los anticipos otorgados por este medio.

\section{Rentabilidad Costo Beneficio}

Después de realizar el análisis de costo Beneficio con los datos encontrados, se puede decir que los tres centros de pago son rentables siendo el más rentable el centro de pago de Somoto, al calcular la rentabilidad anual esta fue de 2.09, para el centro de pago de Sébaco fue de 1.60 y Para Ocotal fue de 1.82, Sin embargo al revisar se puede apreciar con los datos proporcionado que hay gastos que no fueron contabilizados como los gastos de agua, Pago de INSS, IR, INATEC, Teléfono, servicio de Limpieza, esto debido a que son contabilizados en el centro de pago de Estelí, lo cual no es correcto pues se deben de incluir en donde se gasta para obtener los datos reales.

El Administrador en su entrevista informó que cuando el contratante le ofreció que asumirá los servicios de ventanilla en los centros de pagos, no se realizo este análisis, puesto que no llevan una contabilidad actualizada y pueden estar teniendo perdidas en vez de ganancia a como lo valoran actualmente

\section{CONCLUSIONES}

Después de haber analizado los resultados obtenidos en la evaluación del control interno se concluye lo siguiente:

- En lo que respecta al ambiente de control se encuentra una mala segregación de funciones, la que provoca que no se detecten errores involuntarios, omisiones y exponiéndose a actos ilícitos, debido a que la misma persona realiza la recepción de los pagos de las facturas por parte de los clientes, es la encargada de realizar los depósitos, no se realizan arqueos sorpresivos 
- El tener una misión, visión escrita permite que la empresa cumplan con sus objetivos y metas planteados pero el hecho de que los empleados la desconozcan se puede decir que los empleados no está en caminados con los objetivos por qué no los conoce, también se debe dar a conocer la una estructura organizativa sus empleados por lo que la empresa está reduciendo la capacidad de establecer sistemas operacionales que le permitan manejar y controlar con profesionalismo, y así mismo a la toma de decisiones

- La asignación y responsabilidad cada quien sabe cuáles son sus funciones que tiene asignada, las decisiones más importantes se hacen en conjunto y quedan registrada, sin embargo no tienen un manual de funciones escrito. Esto es fundamental para lograr un compromiso mayor en las personas que se desempeñan en una organización.

- En cuanto a la evaluación de riesgos no tienen establecido un procedimiento y no existe control total, el exceso de confianza que se tiene puede ser más fácil el riesgo de fraude lo que puede perjudicar económicamente a la empresa

- La información financiera, no está al día, y peor aun quien retrasa la información financiera es la misma junta directa de la empresa pues ellos no hacen a tiempo sus rendiciones de cuentas lo que ocasiona que no se puede saber cómo están financieramente.

- La comunicación en la empresa se puede decir que es adecuada a pesar de no estar en sus oficinas se les informa oportunamente, sin embargo algunas decisiones importantes no son conocidas por el personal

- La empresa no tiene ningún método de supervisión en cuanto a lo que respecto a caja chica, caja general, y los centros de pagos por esta falta de control debe contar con las políticas y procedimientos establecidos como respuesta para aceptar, compartir, reducir y fortalecer las áreas para mejorar el funcionamiento de la empresa.

- En el análisis de rentabilidad que llevan este no es el adecuado debido a que se basan en la relación costo beneficio y todos los egresos no están contabilizadas correctamente pues los tienen incluidos en otros centros de pagos, por lo tanto no se sabe correctamente si los centros son rentables para la empresa.

\section{RECOMENDACIONES}

Se recomienda a la gerencia en conjunto con la administración deberá establecer políticas y procedimientos para todas las áreas de trabajo, contratar a un contador que conozca los objetivos de la empresa, para evaluar e implementar nuevos controles que le permitan a la empresa lograr los objetivos deseados.

La administración en conjunto con la Junta deberá de diseñar un control sobre los procedimientos en los puntos de Recaudo que permitan administrar riesgos menores, que pueden ser mitigados a través de controles internos y darlo a conocer a lo inmediato.

Se debe dar a conocer su misión, visión, y objetivos estratégicos será de importancia para establecerse claramente y hacerles saber a los trabajadores quiénes son y a donde quieren llegar, de manera que se trabaje en función de alcanzarlo.

Se debe contabilizar todos los egresos correctamente y calcular la rentabilidad anual de cada centro de pago de manera que puedan tener la información a tiempo y tomar las decisiones correctas. 


\section{BIBLIOGRAFÍA}

Arens, Alvin A, Elder, Randal J; Beasley ark S. (2007). Auditoria un Enfoque Integral. México: Editorial Pearson Educación.

Benjamín Franklin, Enrique (2007). Auditoría Administrativa Gestión Estratégica del Cambio, Segunda Edición, México: Pearson Educación.

Catácora, F. (1996). Sistemas y Procedimientos Contables, Primera Edición. Editorial McGraw/Hill. Venezuela.

Cepeda A., Gustavo (1997). Auditoría y Control interno, Primera Edición, Colombia: Editorial Nomos S. A.

Coopers y Lybrand (1997). Los nuevos conceptos del control interno (Informe Coso). Ediciones Díaz de Santos S.A. España.

Estupian Gaitab, Rodrigo (2002)Control Interno y Fraudes, Primera Edición, Bogota Eco Ediciones.

Fonseca Luna, Oswaldo (2011). Sistemas de control interno para organizaciones, Primera Edición, Imprenta Publicidad \&Matiz Lima Perú 2011.

Gironella Masgrau y Velasco auditores. S.A. (2003). Cálculo de la rentabilidad económica de una empresa, Sitio web: Http://www.economistas. org/ECIF/gestor/boletines/Boletin16/03.pdf

Mantilla B, Samuel Alberto. (2005). Control Interno Informe Coso, Cuarta Edición, Colombia, Editorial ECOE EDICIONES.

Rojas Diaz, Walter Orlando (2007). "Tesis

Diseño de un Sistema de Control Interno en una Empresa Comercial, Universidad de San Carlos de Guatemala, Facultad de Ciencias Económicas.

Rusenas, Rubén Oscar (1999). Manual De Control Interno. Ediciones Macchi. Primera Edición. Buenos Aires, Argentina.

Santilla G., Juan R. (2002). Auditoría Interna Integral Administrativa Operacional. Editorial Thonson. México.

Sotomayor, Alfonso Amador (2008).Auditoria Administrativa. Primera Edición. Editorial McGraw Hill. México 